\title{
Quantum transport in high-quality shallow InSb quantum wells
}

\author{
Zijin Lei ${ }^{\dagger}, 1$, a) Christian A. Lehner ${ }^{\dagger},{ }^{1}$ Erik Cheah, ${ }^{1}$ Matija Karalic, ${ }^{1}$ Christopher Mittag, ${ }^{1}$ Luca Alt, ${ }^{1}$ Jan \\ Scharnetzky, ${ }^{1}$ Werner Wegscheider, ${ }^{1}$ Thomas Ihn, ${ }^{1}$ and Klaus Ensslin ${ }^{1}$ \\ Solid State Physics Laboratory, Department of Physics, ETH Zurich, 8093 Zurich, Switzerland
}

(Dated: 26 June 2019)

$\dagger$ These authors contributed equally to this work.

$\mathrm{InSb}$ is one of the promising candidates to realize a topological state through proximity induced superconductivity in a material with strong spin-orbit interactions. In two-dimensional systems, thin barriers are needed to allow strong coupling between superconductors and semiconductors. However, it is still challenging to obtain a high-quality InSb two-dimensional electron gas in quantum wells close to the surface. Here we report on a molecular beam epitaxy grown heterostructure of InSb quantum wells with substrate-side Si-doping and ultra-thin InAlSb (5 nm, $25 \mathrm{~nm}$, and $50 \mathrm{~nm}$ ) barriers to the surface. We demonstrate that the carrier densities in these quantum wells are gate-tunable and electron mobilities up to $350,000 \mathrm{~cm}^{2}(\mathrm{Vs})^{-1}$ are extracted from magneto-transport measurements. Furthermore, from temperature-dependent magneto-resistance measurements, we extract an effective mass of $0.02 m_{0}$ and find a Zeeman splitting compatible with the expected band edge g-factor.

InSb elicits special interest in electronic ${ }^{112}$, electro-optical ${ }^{3}$, and spintronic ${ }^{4}$ applications due to its unique and extreme properties compared to other binary III-V compound semiconductors. Apart from the small band gap and electron effective mass, InSb is considered a candidate for the fabrication of topological quantum devices owing to its strong Rashba spin-orbit interaction (SOI) ${ }^{\sqrt[5]{4}}$ and its intrinsic giant band edge g-factor of $|g| \sim 51 \frac{89}{}$. As proposed by Y. Oreg et al $\stackrel{10}{10}$ a topological superconducting phase can be induced in a one-dimensional semiconductor with strong Rashba SOI in a Zeeman field by the coupling to an s-wave superconductor. Reports in this regard have been published for InAs 11 and $\mathrm{InSb}^{12}$ nanowire-based Majorana devices. In contrast to nanowires, two-dimensional electron gas (2DEG) systems are far more versatile for topological applications. Various types of scalable superconductor-semiconductor hybrid devices have been proposed $13-15$ and experimental research in Al-InAs heterostructures $\frac{16}{18}$ has hence followed. However, despite the superior intrinsic material properties, the progress on InSb 2DEGs is still hampered. Recently, there are developments in free-standing InSb nanostructures and their transport measurements, such as nanosails $\frac{19}{19}$ and nanosheets 20.22 . These layered InSb structures have advantages to achieve direct metal/superconductor contacts on them. Nevertheless, the research on InSb quantum wells (QWs) is still lacking due to the difficulties with heterostructure growth, although QWs have significant potential leading to high-quality devices $23 \mid 24$. In QW systems, a thin barrier is required to induce superconductivity in the 2DEG through the proximity effect. However, the closeness of the 2DEG to the surface can limit the mobility of the carriers as a consequence. In this work, we present a quantum transport experiment, where the InSb 2DEG is close to the sample surface. Our magneto-transport measurements show that the 2DEGs still preserve a high mobility, even for the case of a QW with a barrier to the surface of only $5 \mathrm{~nm}$. We also investigate other unique characteristics of InSb, such as the light electron effective mass $m^{*}$ and the large band edge

\footnotetext{
${ }^{a)}$ Electronic mail: zilei@ phys.ethz.ch
}

g-factor.

We report the fabrication and characterization of three $\mathrm{InSb}$ QW samples doped on the substrate side, which are grown on (100) GaAs substrates by molecular beam epitaxy (MBE). Two schematic layer sequences are shown in Figs. 11(a) and (b). The growth details introduced in Ref 25 are only briefly outlined here. To overcome the lattice mismatch between $\mathrm{GaAs}$ and InSb, an interfacial misfit GaSb buffer as well as an interlayer InAlSb buffer are employed. The total thickness of this buffer system amounts to roughly $3 \mu \mathrm{m}$. The $21 \mathrm{~nm}$-thin InSb QWs are then surrounded by $\operatorname{In}_{0.9} \mathrm{Al}_{0.1} \mathrm{Sb}$ confinement barriers, while n-type carriers are introduced to the active region by a $\mathrm{Si} \delta$-doping layer incorporated $30 \mathrm{~nm}$ below the QW in the barrier on the substrate side. On top of the QW, the $\mathrm{In}_{0.9} \mathrm{Al}_{0.1} \mathrm{Sb}$ layer thicknesses for samples 1 and 2 are $50 \mathrm{~nm}$ and $5 \mathrm{~nm}$, respectively. Sample 1 is the only sample entailing a $10 \mathrm{~nm}$ InSb capping layer, while for samples 2 and $3(25 \mathrm{~nm}$ thick upper barrier) the structure ends with $\operatorname{In}_{0.9} \mathrm{Al}_{0.1} \mathrm{Sb}$ [see Fig. 1(b)]. We adopt the asymmetric bottom-doping scheme to avoid screening of the top gate electric field by the doping layer. A self-consistent band structure simulation of these 3 samples are introduced in the supplementary materiel.

Using wet chemical etching, standard Hall bar structures are defined with an etch depth of more than $120 \mathrm{~nm}$, which is thus deeper than the Si-doping layer. Hall bar samples 1 and 2 have lateral dimensions of $50 \times 25 \mu \mathrm{m}^{2}$ (contact separation $\times$ width) while sample 3 is $10 \times 4 \mu \mathrm{m}^{2}$ in size. Layers of $\mathrm{Ge} / \mathrm{Ni} / \mathrm{Au}$ evaporated on the contact areas of the samples after an Ar sputtering process provide Ohmic contacts to the 2DEG without the necessity of annealing 26 . The samples are coated with a $40 \mathrm{~nm}$-thick aluminium oxide (ALO) dielectric layer using atomic layer deposition at a temperature of $150{ }^{\circ} \mathrm{C}$. Finally, Ti/Au top gates covering the Hall bars are evaporated using electron beam evaporation. As a comparison, van der Pauw geometry samples without chemical etching and ALO are fabricated from the same wafers of the Hall bar samples. Their magneto-transport measurement is introduced in the supplementary material.

The magneto-transport characterization is performed with standard low frequency $(12 \mathrm{~Hz})$ lock-in techniques at a tem- 


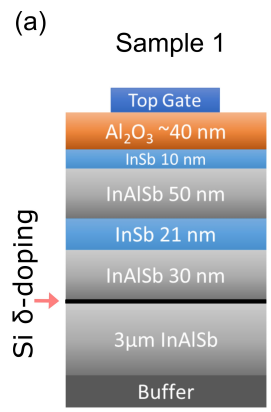

(b)
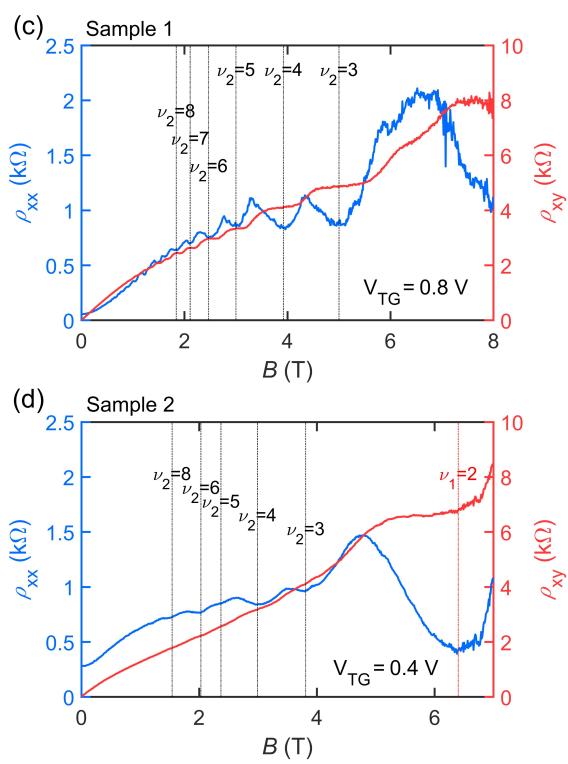

FIG. 1. (a) Layer structure of sample 1. (b) Layer structure of sample 2. The $B$-dependence of $\rho_{x x}$ (blue) and $\rho_{x y}$ (red) of sample 1 and sample 2 are shown in (c) and (d), respectively. The filling factor of the electrons in the doping layer, $v_{1}$, and the filling factor of the electrons in the QW, $v_{2}$, are determined from the $\mathrm{SdH}$ data.

perature of $1.3 \mathrm{~K}$. Figs. 1 (c) and (d) show the dependence of the longitudinal and transverse resistivities $\rho_{x x}$ and $\rho_{x y}$ of samples 1 and 2 in a magnetic field $B$ applied normal to the QW plane, where the top gate voltage $V_{T G}$ are $0.8 \mathrm{~V}$ and 0.4 $\mathrm{V}$ respectively. Shubinikov-de Haas oscillations in $\rho_{x x}$ and plateaus in $\rho_{x y}$ can be seen. In addition, the positive magnetoresistance and the nonlinear Hall resistance found in both samples at fields below about $4 \mathrm{~T}$ imply the existence of parallel conducting channels with distinct mobilities. Thus, the plateaus in $\rho_{x y}$ are not quantized at the expected values of the single-subband quantum Hall effect. The origin of the parallel channels is discussed below.

In the following, we describe the properties of sample 2 in detail, as it comprises the thinnest barrier. It is, therefore, most interesting with regard to a superconducting proximity effect induced by a superconducting contact. Details of samples 1 and 3 are found in the supplementary material. Figs.2 (a) and (b) show $\rho_{x x}$ and $\rho_{x y}$ for sample 2 as a function of the top gate voltage $V_{\mathrm{TG}}$ and the magnetic field $\mathrm{B}$, respectively. Fig. 2(a)
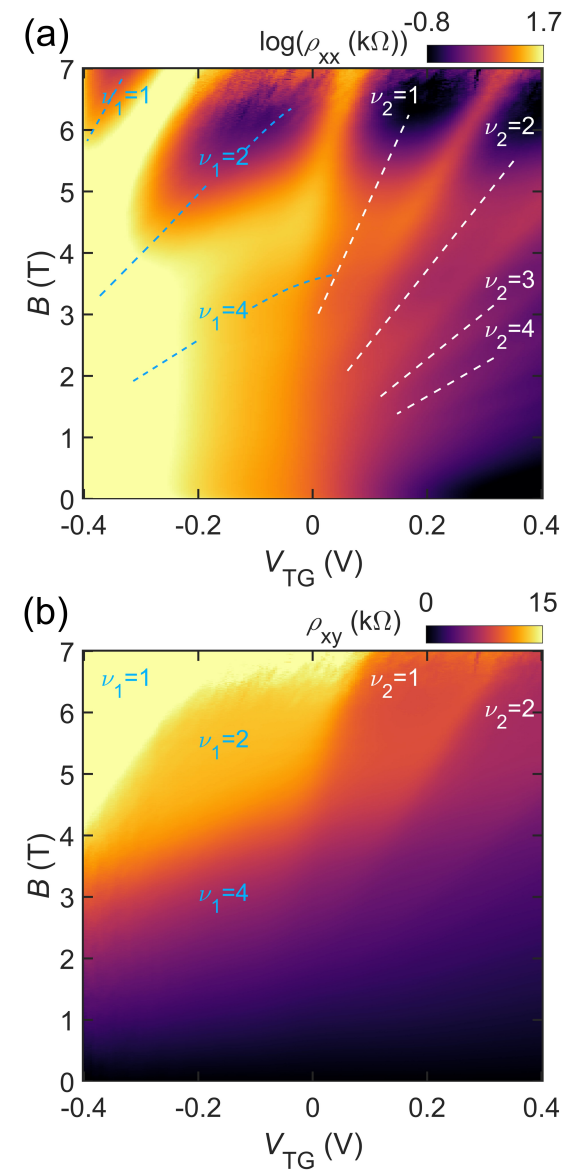

FIG. 2. The detailed transport characterization of sample 2 at $1.3 \mathrm{~K}$ with $\rho_{x x}$ (a) and $\rho_{x y}$ (b) as functions of $V_{\mathrm{TG}}$ and $B$. The Landau fan diagrams and filling factors of the electrons in doping layer and QW layer are labeled with blue and white dashed lines respectively.

reveals two Landau fan diagrams, the first of which appears at low $V_{\mathrm{TG}}$ and is marked with blue dashed lines, while the second Landau fan diagram at high $V_{\mathrm{TG}}$ is marked with white dashed lines. The double-fan structure confirms the presence of two parallel conducting channels in the heterostructure. We extract their carrier densities $n_{1}$ and $n_{2}$ from the $1 / B$ periodicity of the SdH oscillations. As shown in Fig. 3 (a), at lower $V_{\mathrm{TG}}$ only the first channel is populated and the density $n_{1}$ increases linearly with increasing $V_{\mathrm{TG}}$. When $V_{\mathrm{TG}}>-0.1 \mathrm{~V}$, the increase in $n_{1}$ saturates while the second channel gets populated and $n_{2}$ increases linearly instead. The gate capacitances of the first and the second channels are estimated to be $C_{1}=0.7$ $\mathrm{mF} / \mathrm{m}^{2}$ and $C_{2}=1.02 \mathrm{mF} / \mathrm{m}^{2}$, respectively. We attribute $n_{1}$ to carriers in the Si-doping layer and $n_{2}$ to carriers in the $\mathrm{QW}$. The calculated capacitances are within a factor of two of what is expected by considering the layer thicknesses and dielectric constants. The saturation of $n_{1}$ is due to screening of the gate electric field by the electrons populating the QW.

A two-band Drude model allows us to estimate the mobilities $\mu_{1}$ and $\mu_{2}$ of the $\mathrm{Si}$ and the QW layer electrons. Using $n_{1}$ 

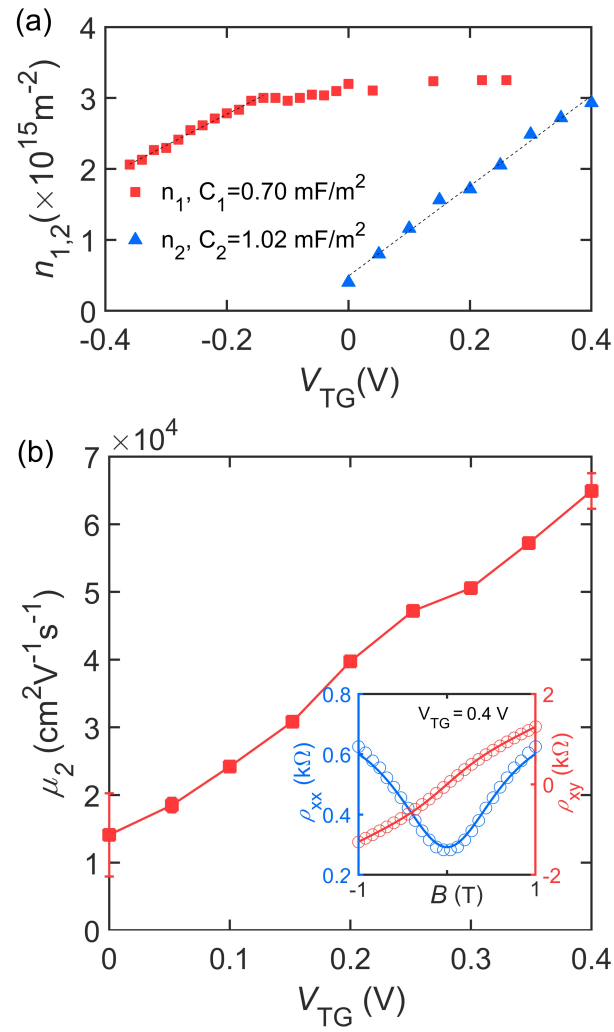

FIG. 3. Analysis of the data shown in Fig. 2 (a) Carrier densities of the two conductive channels vs. $V_{\mathrm{TG}}$. (b) The mobility $\mu_{2}$ extracted from the two-band model vs. $V_{\mathrm{TG}}$. Insert: the data (circles) and fitting (lines) of $\rho_{x x}$ (red) and $\rho_{x y}$ (blue) vs. $B$ when $V_{\mathrm{TG}}=0.4 \mathrm{~V}$.

and $n_{2}$ obtained from the $\mathrm{SdH}$ oscillations, only the two mobilities remain as fitting parameters. As shown in Fig. 3 b), the mobility $\mu_{2}$ increases with the increase of $V_{\mathrm{TG}}$. The insert of Fig. 3.b) shows data (circles) and fitted curves (lines) of the low field $\rho_{x x}$ and $\rho_{x y}$ of sample 2 at a gate voltage $V_{\mathrm{TG}}=0.4 \mathrm{~V}$ in a small magnetic field range. With $n_{1}=3 \times 10^{15} \mathrm{~m}^{-2}$ and $n_{2}=3 \times 10^{15} \mathrm{~m}^{-2}$, we find that mobilities are $\mu_{1}=7,500$ $\mathrm{cm}^{2}(\mathrm{Vs})^{-1}$ and $\mu_{2}=67,000 \mathrm{~cm}^{2}(\mathrm{Vs})^{-1}$, respectively. Phenomenologically, samples 1 and 3 behave similarly and their densities and mobilities are listed in Table 1. Especially, compared with previous related publications 5|923]24[27-30, sample 1 still holds similar or higher mobility of the 2DEG with comparable or thinner barrier thickness.

From temperature-dependent $\mathrm{SdH}$ oscillations the effective mass of the electrons in the InSb QWs can be determined. Fig. 4(a) shows the corresponding measurements for sample 2 with $n_{2}=3.8 \times 10^{15} \mathrm{~m}^{-2}$ determined from the $1 / B$-periodicity. The oscillations of the resistivity $\Delta \rho_{x x}$ are obtained from subtracting the smooth background of the magneto-resistance $\bar{\rho}_{x x} 31$. Fig. 4 (b) shows fits of the Dingle factor ${ }^{32}$ to $\ln \left(\rho_{\mathrm{xx}} / \bar{\rho}_{\mathrm{xx}}\right)$. The obtained effective mass is $m^{*} \approx 0.019 m_{0}$, where $m_{0}$ is the electron mass in vacuum. Using the same method, we find that the effective mass is
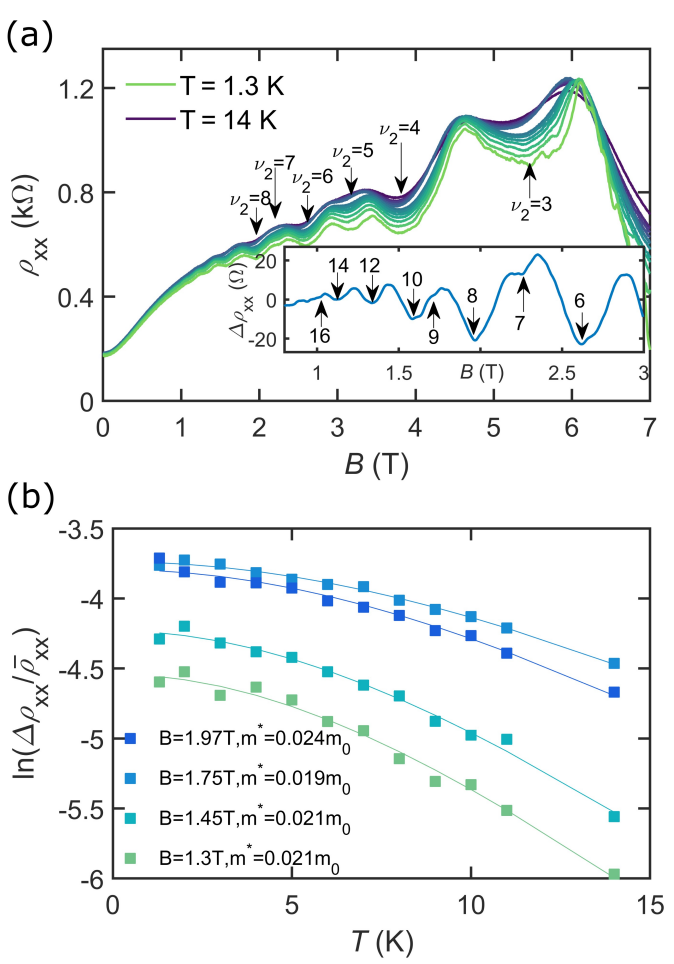

FIG. 4. Effective mass measurement and the Zeeman splitting of sample 2. (a) Temperature-dependence of $\mathrm{SdH}$ oscillations with $n_{2}=$ $3.8 \times 10^{15} \mathrm{~m}^{-2}$. Insert: $\Delta \rho_{x x}$ in a small magnetic field measured at $1.3 \mathrm{~K}$. The Zeeman splitting happens at around $v_{2}=9$. (b) Dingle factor fitting with different $B$. The squares are data and the lines are fitted curves.

TABLE I. Summary of carrier densities, mobilities and effective masses of all the three samples in this work.

\begin{tabular}{cccc}
\hline \hline Properties & Sample 1 & Sample 2 & Sample 3 \\
\hline Upper barrier thickness & $50 \mathrm{~nm}$ & $5 \mathrm{~nm}$ & $25 \mathrm{~nm}$ \\
$\mu_{2(\max x}\left(\mathrm{cm}^{2}(\mathrm{Vs})^{-1}\right)$ & 350,000 & 67,000 & 160,000 \\
$n_{2}\left(\times 10^{15} \mathrm{~m}^{-2}\right)$ & $0-3.5$ & $0-3$ & $0-3$ \\
$m^{*}$ & $0.020 m_{0}$ & $0.019 \pm 0.02 m_{0}$ & - \\
\hline \hline
\end{tabular}

density-independent within the range between $1.8 \times 10^{15} \mathrm{~m}^{-2}$ and $3.8 \times 10^{15} \mathrm{~m}^{-2}$. This result consist with the recent work by Ke et al. 24 .

The spin-splitting of Landau levels is observed at magnetic fields $2 \mathrm{~T}$, as shown in the inset of Fig. (4).a). With increasing $B$, the integer filling factor sequence changes from even to even and odd numbers. The magnetic field value beyond which the spin-splitting is resolved in the experiment is consistent with the band edge g-factor values determined in similar InSb

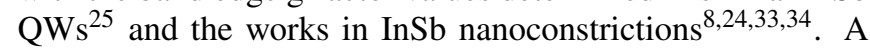
very rough estimate of the band edge g-factor for our device is found in the supplementary material. 
In summary, we have presented an InSb QW hetreostructure with inverted doping and ultra-thin InAlSb barriers to the sample surface. Using a standard Hall bar geometry, we performed magneto-transport measurements and found that the InSb QWs still show tunable densities and high mobilities, despite the small barrier thickness. The parallel conducting channel induced by the Si doping found in the investigated samples can be eliminated by reducing the doping concentration in future devices. We determined the effective in-plane mass of electrons in the InSb QWs to be $0.019 m_{0}$ and estimated a large band edge g-factor close to the intrinsic bulk value. We anticipate that this work paves the way for realizing in-plane InSb devices with a superconducting top gate in the future, where Andreev reflection and 2D Majorana physics may be investigated.

\section{SUPPLEMENTARY MATERIAL}

See supplementary materials for the band edge g-factor estimation, the self-consistent band structure simulation, and the magneto-transport measurements of sample 1, sample 3 and the van der Pauw geometry samples.

\section{ACKNOWLEDGMENTS}

This work was supported by the Swiss National Science Foundation through the National Center of Competence in $\mathrm{Re}$ search (NCCR) Quantum Science and Technology.

${ }^{1}$ T. Ashley, A. Dean, C. Elliott, G. Pryce, A. Johnson, and H. Willis, "Uncooled high-speed InSb field-effect transistors," Applied physics letters 66, 481-483 (1995).

${ }^{2}$ J. Orr, P. Buckle, M. Fearn, C. Storey, L. Buckle, and T. Ashley, “A surfacegated insb quantum well single electron transistor," New Journal of Physics 9, 261 (2007).

${ }^{3}$ H. Chen, J. Heremans, J. Peters, A. Govorov, N. Goel, S. Chung, and M. Santos, "Spin-polarized reflection in a two-dimensional electron system," Applied Physics Letters 86, 032113 (2005).

${ }^{4}$ I. Žutić, J. Fabian, and S. D. Sarma, "Spintronics: Fundamentals and applications," Reviews of modern physics 76, 323 (2004).

${ }^{5}$ M. Leontiadou, K. Litvinenko, A. Gilbertson, C. Pidgeon, W. Branford, L. Cohen, M. Fearn, T. Ashley, M. Emeny, B. Murdin, et al., "Experimental determination of the Rashba coefficient in InSb/InAlSb quantum wells at zero magnetic field and elevated temperatures," Journal of Physics: Condensed Matter 23, 035801 (2011).

${ }^{6}$ R. Kallaher, J. Heremans, N. Goel, S. Chung, and M. Santos, "Spin-orbit interaction determined by antilocalization in an InSb quantum well," Physical Review B 81, 075303 (2010).

${ }^{7}$ G. Khodaparast, R. Doezema, S. Chung, K. Goldammer, and M. Santos, "Spectroscopy of rashba spin splitting in insb quantum wells," Physical Review B 70, 155322 (2004).

${ }^{8}$ F. Qu, J. van Veen, F. K. de Vries, A. J. Beukman, M. Wimmer, W. Yi, A. A. Kiselev, B.-M. Nguyen, M. Sokolich, M. J. Manfra, et al., "Quantized conductance and large g-factor anisotropy in insb quantum point contacts," Nano letters 16, 7509-7513 (2016).

${ }^{9}$ A. Gilbertson, W. Branford, M. Fearn, L. Buckle, P. D. Buckle, T. Ashley, and L. Cohen, "Zero-field spin splitting and spin-dependent broadening in high-mobility $\mathrm{InSb} / \mathrm{In}_{1-x} \mathrm{Al}_{x} \mathrm{Sb}$ asymmetric quantum well heterostructures," Physical Review B 79, 235333 (2009).

${ }^{10}$ Y. Oreg, G. Refael, and F. von Oppen, "Helical liquids and majorana bound states in quantum wires," Physical review letters 105, 177002 (2010).

${ }^{11}$ M. Deng, S. Vaitiekenas, E. B. Hansen, J. Danon, M. Leijnse, K. Flensberg, J. Nygård, P. Krogstrup, and C. M. Marcus, "Majorana bound state in a coupled quantum-dot hybrid-nanowire system," Science 354, 1557-1562 (2016).
${ }^{12}$ H. Zhang, C.-X. Liu, S. Gazibegovic, D. Xu, J. A. Logan, G. Wang, N. Van Loo, J. D. Bommer, M. W. De Moor, D. Car, et al., "Quantized majorana conductance," Nature 556, 74 (2018).

${ }^{13}$ R. P. Riwar, M. Houzet, J. S. Meyer, and Y. V. Nazarov, "Multi-terminal Josephson junctions as topological matter,' Nature Communications 7, 1-5 (2016)

${ }^{14}$ A. Stern and E. Berg, "Fractional Josephson vortices and braiding of majorana zero modes in planar superconductor-semiconductor heterostructures," Physical Review Letters 122, 107701 (2019).

${ }^{15}$ Y. Peng, F. Pientka, E. Berg, Y. Oreg, and F. von Oppen, "Signatures of topological josephson junctions," Phys. Rev. B 94, 085409 (2016)

${ }^{16}$ H. J. Suominen, M. Kjaergaard, A. R. Hamilton, J. Shabani, C. J. Palmstrøm, C. M. Marcus, and F. Nichele, "Zero-energy modes from coalescing andreev states in a two-dimensional semiconductor-superconductor hybrid platform,' Phys. Rev. Lett. 119, 176805 (2017)

${ }^{17}$ A. Fornieri, A. M. Whiticar, F. Setiawan, E. Portolés, A. C. Drachmann, A. Keselman, S. Gronin, C. Thomas, T. Wang, R. Kallaher, et al., "Evidence of topological superconductivity in planar josephson junctions," Nature , 1 (2019).

${ }^{18}$ A. Whiticar, A. Fornieri, E. O'Farrell, A. Drachmann, T. Wang, C. Thomas, S. Gronin, R. Kallaher, G. Gardner, M. Manfra, et al., "Interferometry and coherent single-electron transport through hybrid superconductorsemiconductor coulomb islands," arXiv preprint arXiv:1902.07085 (2019).

${ }^{19}$ M. de la Mata, R. Leturcq, S. R. Plissard, C. Rolland, C. Magen, J. Arbiol, and P. Caroff, "Twin-induced InSb nanosails: a convenient high mobility quantum system," Nano letters 16, 825-833 (2016).

${ }^{20}$ D. Pan, D. Fan, N. Kang, J. Zhi, X. Yu, H. Xu, and J. Zhao, "Free-standing two-dimensional single-crystalline InSb nanosheets," Nano letters 16, 834841 (2016).

${ }^{21}$ N. Kang, D. Fan, J. Zhi, D. Pan, S. Li, C. Wang, J. Guo, J. Zhao, and H. Xu, "Two-dimensional quantum transport in free-standing InSb nanosheets," Nano letters 19, 561-569 (2018).

${ }^{22}$ J. Xue, Y. Chen, D. Pan, J.-Y. Wang, J. Zhao, S. Huang, and H. Xu, "Gate defined quantum dot realized in a single crystalline InSb nanosheet," Applied Physics Letters 114, 023108 (2019).

${ }^{23}$ W. Yi, A. A. Kiselev, J. Thorp, R. Noah, B.-M. Nguyen, S. Bui, R. D. Rajavel, T. Hussain, M. F. Gyure, P. Kratz, et al., "Gate-tunable high mobility remote-doped $\mathrm{InSb} / \mathrm{In}_{1-x} \mathrm{Al}_{x} \mathrm{Sb}$ quantum well heterostructures," Applied Physics Letters 106, 142103 (2015).

${ }^{24}$ C. T. Ke, C. M. Moehle, F. K. de Vries, C. Thomas, S. Metti, C. R. Guinn, R. Kallaher, M. Lodari, G. Scappucci, T. Wang, et al., "Ballistic superconductivity and tunable $\pi$-junctions in InSb quantum wells," arXiv preprint arXiv:1902.10742 (2019).

${ }^{25}$ C. A. Lehner, T. Tschirky, T. Ihn, W. Dietsche, J. Keller, S. Fält, and W. Wegscheider, "Limiting scattering processes in high-mobility InSb quantum wells grown on GaSb buffer systems," Phys. Rev. Materials 2, 054601 (2018)

${ }^{26}$ N. Goel, J. Graham, J. Keay, K. Suzuki, S. Miyashita, M. Santos, and Y. Hirayama, "Ballistic transport in InSb mesoscopic structures," Physica E: Low-dimensional Systems and Nanostructures 26, 455 - 459 (2005) international Conference on Quantum Dots.

${ }^{27}$ O. Pooley, A. Gilbertson, P. Buckle, R. Hall, M. Emeny, M. Fearn, M. Halsall, L. Cohen, and T. Ashley, "Quantum well mobility and the effect of gate dielectrics in remote doped $\mathrm{InSb} / \mathrm{Al}_{x} \mathrm{In}_{1-x} \mathrm{Sb}$ heterostructures," Semiconductor Science and Technology 25, 125005 (2010).

${ }^{28}$ F. Gouider, Y. B. Vasilyev, M. Bugár, J. Könemann, P. Buckle, and G. Nachtwei, "Terahertz photoresponse of alinsb/insb/alinsb quantum well structures," Physical Review B 81, 155304 (2010).

${ }^{29}$ M. Uddin, H. Liu, K. Yang, K. Nagase, T. Mishima, M. Santos, and Y. Hirayama, "Characterization of insb quantum wells with atomic layer deposited gate dielectrics," Applied Physics Letters 101, 233503 (2012).

${ }^{30}$ J. Mlack, K. Wickramasinghe, T. Mishima, M. Santos, and C. Marcus, "Inplane magnetoconductance mapping of insb quantum wells," arXiv preprint arXiv:1902.07570 (2019).

${ }^{31}$ B. Habib, M. Shayegan, and R. Winkler, "Spin-orbit interaction and transport in GaAs two-dimensional holes," Semiconductor Science and Technology 23, 1 (2009)

${ }^{32}$ T. Ihn, Semiconductor Nanostructures: Quantum states and electronic transport (Oxford University Press, 2010).

${ }^{33}$ H. A. Nilsson, P. Caroff, C. Thelander, M. Larsson, J. B. Wagner, L.-E. 
Wernersson, L. Samuelson, and H. Xu, "Giant, level-dependent g factors in InSb nanowire quantum dots," Nano letters 9, 3151-3156 (2009).

${ }^{34}$ H. Nilsson, O. Karlström, M. Larsson, P. Caroff, J. N. Pedersen, L. Samuel- son, A. Wacker, L.-E. Wernersson, and H. Xu, "Correlation-induced conductance suppression at level degeneracy in a quantum dot," Physical review letters 104, 186804 (2010). 